\title{
Disentería y absceso hepático en el Chile colonial y republicano. El Doctor Miguel Claro Vásquez
}

\author{
Enrique Laval R.
}

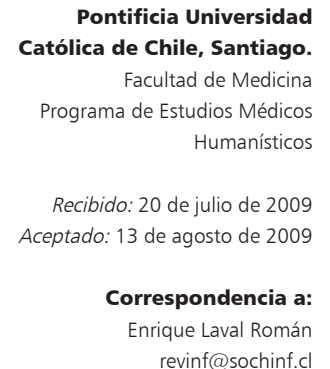

revinf@sochinf.cl

\section{Disentery and hepatic abscess in the colonial and republican Chile. Doctor Miguel Claro Vásquez}

The frequency and severity of dysentery and hepatic abscess during the colonial and republican era in Chile are reviewed. The amebian etiology was confirmed in both clinical entities. Also, Miguel Claro Vásquez, physician and later priest and bishop of the Catholic Church, was distinguished for his contribution to hepatic abscess surgery.

Key words: Dysentery, hepatic abscess, amebian infection.

Palabras clave: Disentería, absceso del hígado, infección amebiana.

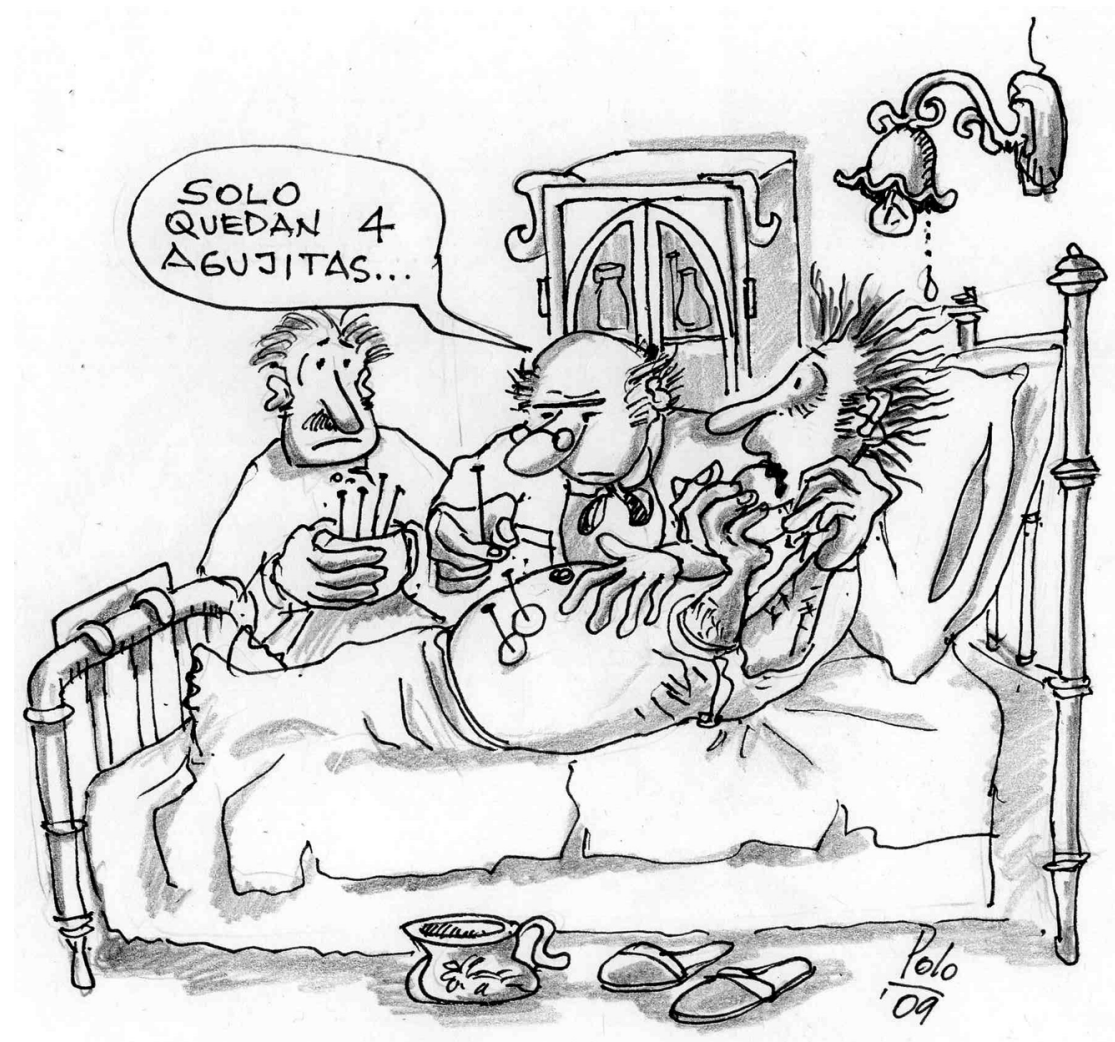

nomía muy característica por su gravedad y gran número, constituyendo una de las calamidades más terribles ${ }^{1}$.

Las epidemias más importantes de disentería en la Colonia correspondieron a los años 1628, 1648, 1668, 1718 y 1773.

El padre Olivares y fray Francisco Menéndez cuentan que en 1706, hubo entre los pehuenches, una "epidemia de cursos de sangre", que según los brujos, fue ocasionada por haber pasado por sus tierras el padre Laguna y una señora española - una imagen de la Virgen traída de Lima para la Misión- lo que produjo muy malos ratos a los padres colonizadores.

En el verano de 1773 hubo una epidemia de disentería en Concepción, lo que motivó a las autoridades gubernamentales solicitar al Protomédico don Ignacio de Jesús Sambrano arbitrar las medidas terapéuticas pertinentes, las que reseñó en un informe emitido en Santiago y que se transcribe textualmente:

" El protomédico de este Reino en obedecimiento del superior decreto de US, que la disentería epidémica que está contagiando la ciudad de Concepción se deve curar de dos modos: primeramente evacuando la causa principal material que corroe y lastima las tripas, esta si biene de la sangre la que desemboca en el intestino duodeno por las arteriolas que visten su túnica, lo que se conoce por padecer asimismo el enfermo una fiebre ardiente, se debe sangrar de loa brazos, las veces necesarias según las fuerzas del paciente. Y, si por esta causa material indigesta contenida en el estómago o mesentereo, se deven curar con Ruibarbo, Crémor tártaro y Jarabe Rosado Resolutivo. Y si en uno u otro caso no obedeciera la enfermedad a los referidos subsidios, se debe ocurrir y valerse de la Ancora especifica, que es la Cartagena, o 
polvos de Ipecacuana confortando de noche para dormir con el Diascordio, Mirabolanos, polvos de coral, tierra sellada en vino, por que tiene por inconseguible en este tiempo el agua destilada de canela. Este es su parecer y juro a Dios y a una cruz proceder con verdad en lo Referido. Santiago y febrero 20 de 1773."

Ignacio de Jesús Sambrano ${ }^{2,3}$.

En 1814, don José Gregorio Paredes médico y cosmógrafo mayor del Perú que se había domiciliado en los alrededores de Santiago por motivos de salud, sobre "las enfermedades observadas en Chile durante 15 meses de residencia", exponía "que las disenterías son muy raras $y$ de marcha más lenta que en Lima". Sin embargo, en otra comunicación del profesor Julio Lafargue, publicada en una revista francesa en 1851, relata que "entre las enfermedades más frecuentes en Chile, se encuentran la disentería, la hepatitis y los abscesos idiopáticos del hígado." Lafargue afirma: "la disentería es un verdadero azote para Chile, donde es endémica, reina todo el año y contribuye tanto a la mortalidad, que por si sola hace más víctimas que las afecciones cerebrales y toráxicas agudas como no las provoca en Europa el cólera, el tifus y todas las gastroenteritis reunidas." Finalmente hace notar "las relaciones de coincidencia y de sucesión que median entre la hepatitis supurativa y la disentería, de tal manera que en los individuos acometidos frecuentemente con la última, se debe sospechar casi siempre el desarrollo latente de la primera."2.

El doctor Wenceslao Díaz recuerda "que en 1826 empezó sus estragos la disentería tropical o gangrenosa y que ahora es más bien una endemia poco generalizada y esporádica en la estación de los grandes cambios atmosféricos o en las épocas en que el pueblo abusa de los alimentos indigestos."

Pero comparando estos informes, el doctor Wenceslao Díaz al ver el inmenso desarrollo que habían tomado las enfermedades del hígado, cuyo punto de partida parecía coincidir con la aparición de la disentería, exclamó: ¡En treinta años operarse un cambio tan grande, tan espantoso que hace presagiar las mayores y más fatales consecuencias! "Algunos de los profesores que me escuchan, han visto efectuarse estas evoluciones a sus propios ojos, han podido seguirlas paso a paso, palpar sus resultados y hoy pueden establecer las más desconsoladoras comparaciones." ${ }^{4}$.

En 1843 y cuando la disentería conservaba todo su carácter alarmante, la Facultad de Medicina, que designaba anualmente un tema sobre las enfermedades más graves y frecuentes en Chile, insistiendo sobre sus características más sobresalientes, eligió como primer tema para la composición que aspirase al premio de 1844, un tratado sobre la disentería con las particularidades que presenta en nuestro país, siendo dividido entre los tres mejores trabajos, imprimiéndose extractados en el primer tomo de los Anales de la Universidad de Chile, correspondiente a los años 1843 y 1844. En los años siguientes aparecieron más de una docena de extensas publicaciones sobre el mismo tema en los Anales. La mayoría fueron Memorias de prueba para optar al grado de licenciado de la Facultad y otras correspondieron a discursos de incorporación de miembros académicos, como los de los doctores Jorge Petit, Wenceslao Díaz y Valentín Saldías, entre otros.

Entre los autores de tesis de grado, destacó nítidamente el doctor Miguel Claro Vásquez, cuya memoria “Observaciones clínicas sobre el tratamiento de los abscesos hepáticos", en 1884, despertó extraordinario interés, siendo uno de los trabajos más importantes de la abundante literatura médica de aquel tiempo sobre el tema ${ }^{1,5}$.

El doctor Claro preconizó en toda sospecha de absceso la punción y en caso confirmado, su vaciamiento quirúrgico, para no exponer al enfermo a los azares de una abertura espontánea; pero el temor al peritoneo, presente en el espíritu de todos los cirujanos de aquellos años retardaba y muchas veces impedía la intervención, por lo que trataban de aislarlo, determinando previamente adherencias. Así, el doctor Miguel Claro puso en práctica un procedimiento que le había sugerido el profesor Ugarte Gutierrez y que describimos textualmente aquí:

"Se escogen seis u ocho alfileres de los usados comúnmente para la sutura esortijada, una pinza porta-agujas se desinfecta convenientemente, asi como el punto donde se va a introducirlos. Se pasa cada alfiler por un trocito de esparadrapo cortado circularmente, que sirve para mantenerlos fijos; colocados en la pinza y previa la anestesia local obtenida por un pulverizador de éter se introducen perpendicularmente hasta penetrar en el foco, a distancia de menos un centímetro uno de otro, describiendo con ellos el trayecto que va a recorrer el bisturí. Se atan los alfileres con un hilo, se adhiere el esparadrapo a la piel y se cubre con una pieza de la misma sustancia. Si durante la operación hemos hecho volver el rostro al enfermo, ni siquiera se apercibe de lo que hemos hecho, pues que la molestia es insignificante."

Los alfileres se dejaban 48 horas y seguro de la existencia de adherencias, se procedía a abrir y a vaciar el absceso. Este método que puso en práctica el doctor Claro fue el preferido de los cirujanos por su comodidad, hasta que largos años más tarde los perfeccionamientos de la antisepsia y de la asepsia les permitieron hacer perder el temor al peritoneo ${ }^{5,6}$.

Muy interesante es el estudio de la evolución gradual del concepto sobre la etiología de la disentería y del absceso hepático, como también de las múltiples ideas y conjeturas que trataban de explicar la frecuente coexistencia de ambas afecciones. La naturaleza íntima de 
Figura 2. Monseñor Miguel Claro, Obispo auxiliar de Santiago de Chile (1919 - 1921).

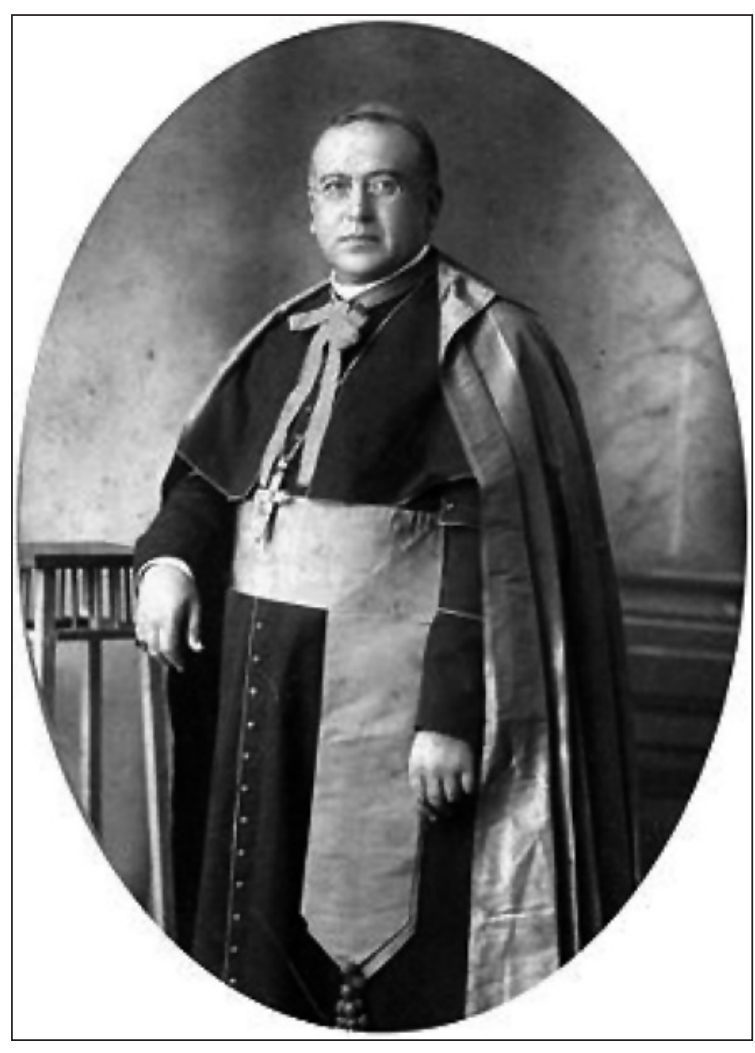

la disentería y del absceso hepático quedó naturalmente desconocida hasta el advenimiento de la parasitología y el descubrimiento de Entamoeba histolytica. En un principio la disentería era considerada como un simple reumatismo intestinal, como una "difteria"de la mucosa intestinal o puramente catarral. Otros autores hablaban de una "alteración primitiva de la bilis por orgasmo del hígado" o sencillamente por una "alteración mórbida especial"1.

La naturaleza parasitaria de la afección había sido sospechada primero por Friedrich Lösch en Rusia (San Petersburgo), en 1875, al demostrar la presencia de amebas en las heces disentéricas y transmitir experimentalmente la disentería al perro. Después Koch y Kartulis, encontraron las mismas formas protozoarias, en las ulceraciones de algunos disentéricos en Egipto e India y también en el pus de un absceso hepático, denominado en esa época "tropical."

Pero fue necesario llegar a 1903 para encontrar un trabajo que proporcionó valiosas aclaraciones sobre este tema. El autor fue el célebre protozoólogo alemán Schaudinn. Este investigador demostró que en el intestino del hombre puede haber dos especies de amebas: una patógena y otra comensal. Publicó una descripción correcta de ambas, como también de su respectiva biología. A la especie patógena la denominó Entamoeba histolytica y a la no patógena Entamoeba coli. Walker y Sellards, demostraron en 1913, la patogenicidad de E. histolytica, en voluntarios humanos ${ }^{7-11}$.

Adolfo Reccius "cree no exagerar, al decir que no ha habido enfermedad en Chile, que haya merecido un estudio más atento e investigación paciente que la disentería amebiana y el absceso hepático. Cuando empezaron a imperar los preceptos de una higiene razonable y a constituirse el tratamiento con la emetina, comenzó también a experimentar una transformación radical la cirugía del absceso hepático amebiano."

Hay pocos ejemplos más notables, en la historia de la medicina, en que el progreso de la medicina general ha reducido el campo y la importancia de la cirugía. Con el tratamiento más oportuno y racional de la colitis amebiana, disminuyó notablemente la localización secundaria en el hígado, de tal manera que dicha complicación se hizo excepcional ${ }^{1}$.

Volvamos al doctor Miguel Claro Vásquez. Había nacido en Santiago de Chile en el año 1861. Después de realizar con brillo sus estudios secundarios en el Seminario Conciliar de Santiago, inició los de Medicina, logrando su título profesional el 12 de enero de 1885.

El doctor Claro estableció su consultorio en la calle Tres Montes (hoy José Miguel de la Barra), ejerciendo con singular éxito la Medicina y Cirugía generales, durante dos años. Pero no era ésta su vocación: la verdadera lo llevaba a la Iglesia, ingresando al Seminario de la Arquidiócesis de Santiago, donde recibió la ordenación sacerdotal en el mes de marzo de 1888. Al principio se desempeñó como capellán de la Hermandad de Dolores, pero luego el Arzobispo don Mariano Casanova lo designó Secretario de la Curia. A partir del 30 de junio de 1902 fue nombrado Vicario General de la Arquidiócesis. En ese elevado cargo demostró condiciones excepcionales, destacando por su competencia, bondad y comprensión humana. Sus más caras preocupaciones las dedicó a la enseñanza y a los problemas de los obreros. Fue el primero en traducir al castellano la Encíclica Rerum Novarum del Papa León XIII y la dio a conocer a los cristianos como nuevas ideas católicas entre los sacerdotes, trabajadores y estudiantes universitarios. Su apostólica vinculación con la cuestión obrera lo llevó a establecer las bases del sindicalismo obrero católico. Fue un estudioso de las Sagradas Escrituras y del Derecho Canónico. La Santa Sede lo elevó al episcopado y el 9 de agosto de 1908 fue nombrado Obispo Titular de Legione. En 1919, el Sumo Pontífice lo designó Obispo Auxiliar de la arquidiócesis de Santiago (Figura 2).

Monseñor Miguel Claro falleció en Santiago el 19 de Mayo de 1921, siendo sepultado en el interior de la Iglesia de la Recoleta Domínica.

Como un homenaje a su memoria se le dio a la calle en que vivió durante largos años el nombre de Miguel 
Claro y su casa ubicada en esa calle esquina de Avenida Providencia, fue posteriormente adquirida por el Gobierno y destinada al funcionamiento del Liceo José Victorino Lastarria.

Monseñor Miguel Claro Vásquez fue el primer obispo médico chileno ${ }^{6,12}$.

\section{Resumen}

En esta comunicación se destaca la frecuencia y gravedad en Chile, durante la época colonial y republicana, de dos entidades clínicas: disentería y absceso hepático, comprobándose finalmente la etiología amebiana en ambos procesos. Además se distingue al doctor Miguel Claro Vásquez, médico, después sacerdote y obispo de la Iglesia Católica, por su aporte a la cirugía del absceso del hígado.

\section{Referencias}

1.- Reccius E A. Historia y desarrollo de la cirugía abdominal en Chile. Ed. Zig-Zag. Santiago de Chile. 1948.
2.- Salas Olano E. Historia de la Medicina en Chile. Impta. Vicuña Mackenna. Santiago de Chile. 1894.

3.- Ferrer P L. Historia de la Medicina en Chile. Tomo I. Impta. Talca. Chile. 1904

4.- Díaz G W. Geografía Médica de Chile. De las enfermedades epidémicas. Rev. Méd. Chile. 1874, 3: 329-52

5.- Claro V M. Observaciones clínicas sobre el tratamiento de los abscesos hepáticos. Rev Méd Chile 1885; 13: 361-83; 401-5.

6.- Laval M E. Noticias sobre los médicos en Chile. Ed. Historia Médica. Santiago de Chile. 1972.

7.- Pedro-Pons A. Patología y Clínica Médicas. Tomo VI. Enfermedades Infecciosas. Ed. Servet. Barcelona. España. 1952.

8.- Veronesi R. Enfermedades Infecciosas y Parasitarias. Ed. Ateneo. Buenos Aires. Rep. Argentina. 1971.

9.- Cruz-Coke E, Lira S G. Estudio de la disentería en Chile. Rev Méd Chile 1932, 60: 173-80.

10.- Alessandri R H, García P, Lerner J. Diagnóstico y tratamiento de las parasitosis intestinales más frecuentes. Rev Méd Chile 1943; 71: 199-211.

11.- García P P, Lerner J. Diagnóstico y tratamiento del absceso hepático amebiano. Rev Méd Chile 1941; 69: 67-78.

12.- http://es.wikipedia.org/wiki/Miguel Claro. (accedido el 24/03/2009). 